\title{
Erratum to: The role of the dorsal hippocampus in two versions of the touchscreen automated paired associates learning (PAL) task for mice
}

\author{
Chi Hun Kim ${ }^{1}$ - Christopher J. Heath ${ }^{1}$ • Brianne A. Kent ${ }^{1}$ - Alexa E. Horner ${ }^{2}$. \\ Timothy J. Bussey ${ }^{1} \cdot$ Lisa M. Saksida ${ }^{1}$
}

Published online: 25 July 2015

(C) Springer-Verlag Berlin Heidelberg 2015

\section{Erratum to: Psychopharmacology}

DOI 10.1007/s00213-015-3949-3

Alexa E. Horner should be listed as the fourth author. Her affiliation is Synome Ltd., Babraham Research Campus, Cambridge, CB22 3AT, UK.

She contributed to the present article in the form of pilot experiments assessing the effect of pre- and post-acquisition lesions of the dorsal hippocampus on dPAL acquisition and performance, respectively. These pilot experiments were a significant catalyst in the conception and design of the studies in the present article. Furthermore, Alexa E. Horner contributed intellectually to the present article in several discussions with the other authors, and approved the final version of the manuscript.

The online version of the original article can be found at http://dx.doi.org/ 10.1007/s00213-015-3949-3.

Chi Hun Kim

chk35@cam.ac.uk

1 Department of Psychology, MRC/Wellcome Trust Behavioural and Clinical Neuroscience Institute, University of Cambridge, Downing St, Cambridge CB2 3EB, UK

2 Synome Ltd., Babraham Research Campus, Cambridge CB22 3AT, UK 